\title{
Bone-Preserving Decompression Procedures Have a Minor Effect on the Flexibility of the Lumbar Spine
}

\author{
Francesco Costa, M.D., Claudia Ottardi, Ph.D., ${ }^{2}$ David Volkheimer, Ph.D., ${ }^{3}$ Alessandro Ortolina, M.D., ${ }^{1}$ Tito Bassani, Ph.D., \\ Hans-Joachim Wilke, Ph.D., ${ }^{3}$ Fabio Galbusera, Ph.D. ${ }^{4}$ \\ Department of Neurosurgery, Humanitas Clinical and Research Center, Rozzano, Italy \\ LaBS, ${ }^{2}$ Department of Chemistry, Material and Chemical Engineering, Politecnico di Milano, Milano, Italy \\ Institute of Orthopaedic Research and Biomechanics, ${ }^{3}$ Trauma Research Centre Ulm, Ulm University, Ulm, Germany \\ IRCCS Galeazzi Orthopedic Institute, ${ }^{4}$ Milano, Italy
}

Objective : To mitigate the risk of iatrogenic instability, new posterior decompression techniques able to preserve musculoskeletal structures have been introduced but never extensively investigated from a biomechanical point of view. This study was aimed to investigate the impact on spinal flexibility caused by a unilateral laminotomy for bilateral decompression, in comparison to the intact condition and a laminectomy with preservation of a bony bridge at the vertebral arch. Secondary aims were to investigate the biomechanical effects of two-level decompression and the quantification of the restoration of stability after posterior fixation.

Methods : A universal spine tester was used to measure the flexibility of six L2-L5 human spine specimens in intact conditions and after decompression and fixation surgeries. An incremental damage protocol was applied : 1) unilateral laminotomy for bilateral decompression at $\mathrm{L} 3-\mathrm{L} 4 ; 2$ ) on three specimens, the unilateral laminotomy was extended to $L 4-\mathrm{L} 5$; 3) laminectomy with preservation of a bony bridge at the vertebral arch (at L3-L4 in the first three specimens and at $L 4-L 5$ in the rest); and 4) pedicle screw fixation at the involved levels.

Results : Unilateral laminotomy for bilateral decompression had a minor influence on the lumbar flexibility. In flexion-extension, the median range of motion increased by $8 \%$. The bone-preserving laminectomy did not cause major changes in spinal flexibility. Two-level decompression approximately induced a twofold destabilization compared to the single-level treatment, with greater effect on the lower level. Posterior fixation reduced the flexibility to values lower than in the intact conditions in all cases.

Conclusion : In vitro testing of human lumbar specimens revealed that unilateral laminotomy for bilateral decompression and bone-preserving laminectomy induced a minor destabilization at the operated level. In absence of other pathological factors (e.g., clinical instability, spondylolisthesis), both techniques appear to be safe from a biomechanical point of view.

Key Words : Laminectomy · Lumbosacral region · Spine · latrogenic disease · Decompression, Surgical · Pedicle screws.

- Received : January 26, 2018 •Revised : February 20, 2018 •Accepted : February 28, 2018

- Address for reprints : Fabio Galbusera, Ph.D.

IRCCS Galeazzi Orthopedic Institute, Via Riccardo Galeazzi 4, Milano 20161, Italy

Tel : +39-02-66214939, Fax : +39-02-66214800, E-mail : fabio.galbusera@grupposandonato.it

This is an Open Access article distributed under the terms of the Creative Commons Attribution Non-Commercial License (http://creativecommons.org/licenses/by-nc/4.0) which permits unrestricted non-commercial use, distribution, and reproduction in any medium, provided the original work is properly cited. 


\section{INTRODUCTION}

Degenerative lumbar stenosis is a narrowing of the spinal canal due to hypertrophy and ossification of the flaval ligament, bulging of the intervertebral disc as well as degenerative alterations of the facet joints ${ }^{23)}$. Lumbar stenosis may provoke pain as well as various neurological symptoms and deficits, such as neurogenic claudication and intermittent pain radiating to the leg, which is exacerbated by prolonged standing or other activities ${ }^{27)}$. The first choice medical treatment of lumbar stenosis is usually conservative ${ }^{10)}$, but decompression surgeries are frequently performed in cases in which medication and physiotherapy do not sufficiently relieve pain and improve function $^{12)}$.

The most consolidated lumbar decompression procedure is wide laminectomy, in which the laminae are removed to enlarge the neural space ${ }^{13,16}$. The procedure is frequently combined with facetectomy, i.e., the additional removal of the facet joints ${ }^{25}$. Despite the high success rate of these techniques in relieving neurological symptoms ${ }^{1,13,22)}$, clinical studies showed that wide laminectomy may provoke iatrogenic instability, therefore requiring concomitant or subsequent fixation and fusion ${ }^{9,18,26)}$. Following the pioneering paper of Allen et al. ${ }^{2)}$, in vitro studies investigating the biomechanics of laminectomy reported coherent results, in particular an increase of spinal flexibility in all motion planes at the operated level after decompression $^{21)}$. In an in vitro study carried out on human specimens, Bisschop et al. ${ }^{4)}$ found an increase of the range of motion (ROM) of 7.3\% in flexion-extension, $7.5 \%$ in lateral bending and $12.2 \%$ in axial rotation after facet-sparing laminectomy. Similar values were found by other authors ${ }^{16,19,32}$. The mechanical strength of the operated level was also found to be significantly reduced ${ }^{3,5)}$.

To mitigate the risk of iatrogenic instability, bone-preserving decompression techniques allowing for a better protection of the spine stability have been introduced ${ }^{7,8)}$. In particular, bilateral laminotomy and unilateral laminotomy for bilateral decompression are widely employed nowadays with good clinical results ${ }^{25)}$. Laminectomy itself, which may be indicated in more severe cases in which a laminotomy may be insufficient to relieve the symptoms, has been refined by preserving a laminar bony bridge. However, despite the abundance of in vitro studies investigating the biomechanical effect of laminectomy, the advantages of bone-preserving techniques in terms of spine flexibility were never extensively investigated. Only a numerical investigation showed a minor effect on the ROM combined with a stress increase in the neural arch after minimally invasive decompression ${ }^{14)}$. Nevertheless, in vitro

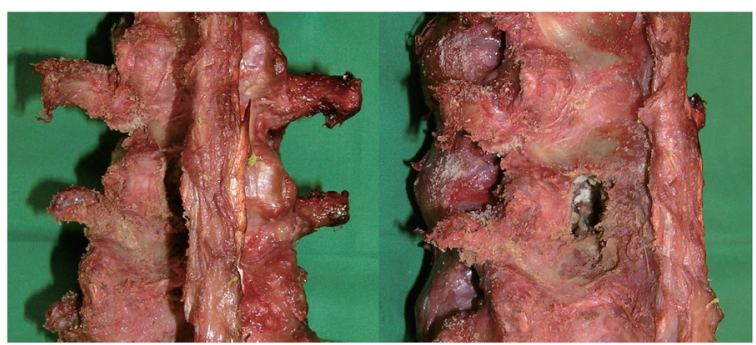

UNI-1L

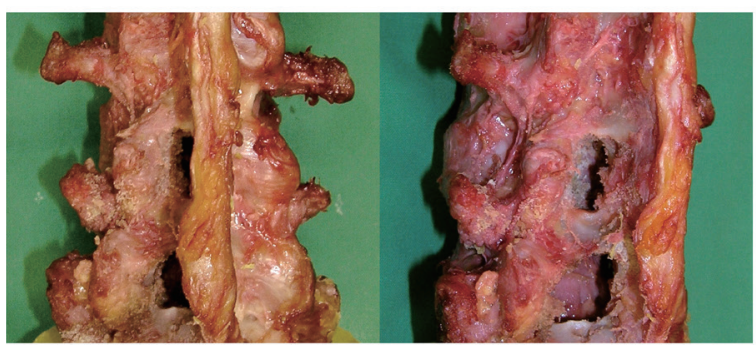

UNI-2L

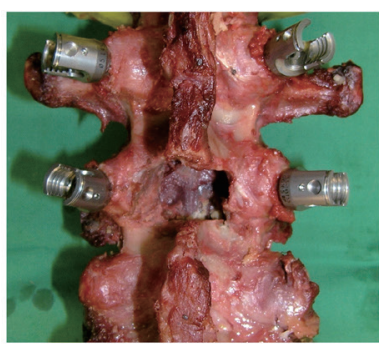

Bl-1L

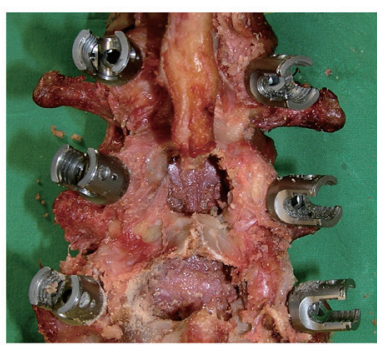

$\mathrm{BI}-2 \mathrm{~L}$

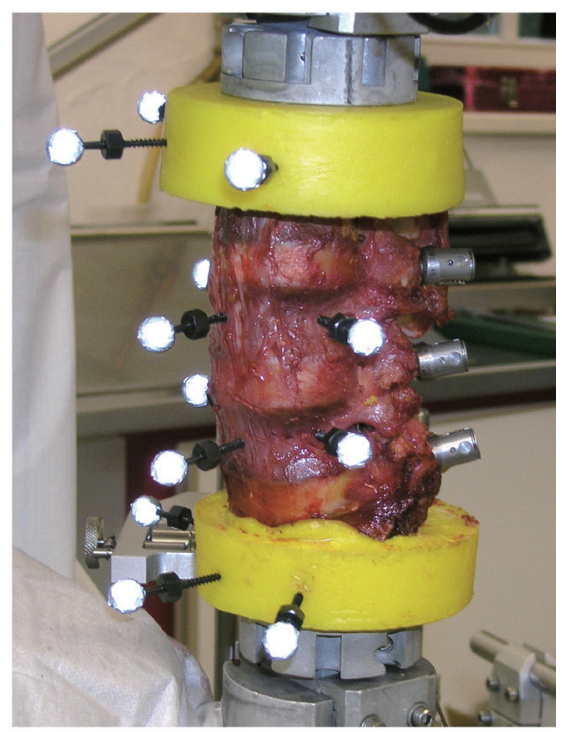

Experimental set-up

Fig. 1. Decompression and stabilization procedures performed on the specimens : unilateral laminotomy for bilateral decompression at L3-L4 (UNI-1L, $\mathrm{n}=6$ specimens); bone-preserving laminectomy at $\mathrm{L} 3-\mathrm{L} 4(\mathrm{BI}-1 \mathrm{~L}, \mathrm{n}=3)$; unilateral laminotomy for bilateral decompression at $\mathrm{L} 3-\mathrm{L} 5(\mathrm{UNI}-2 \mathrm{~L}, \mathrm{n}=3)$; bonepreserving laminectomy at $\mathrm{L} 3-\mathrm{L} 5(\mathrm{BI}-2 \mathrm{~L}, \mathrm{n}=3)$. Experimental set-up used for load application and motion analysis with an optoelectronic motion analysis system (right). 
studies on human lumbar specimens subjected to bone-preserving lumbar decompression were never conducted.

The aim of the present study was therefore to characterize the alterations of the spine flexibility caused by a recent widely used bone-preserving decompression technique, i.e., the unilateral laminotomy for bilateral decompression in comparison to the intact conditions and a refined, bone-preserving laminectomy procedure. Secondary aims were the investigation of the biomechanical effects of two-level decompression techniques as compared with single level surgeries, and the quantification of the restoration of flexibility which may be achieved after posterior fixation with pedicle screws and rods.

\section{MATERIALS AND METHODS}

Six fresh-frozen human lumbar specimens not exhibiting major degenerative conditions (L2-L5; age, 32-68 years; median, 47 years) have been acquired after approval of the relevant ethical committee. The specimens included vertebrae, intervertebral discs and spinal ligaments (anterior longitudinal, posterior longitudinal, flaval, interspinous, supraspinous, capsular, whereas the intertransverse ligament was resected during the harvesting procedure). For all specimens, the proximal and the distal end of L2 and L5 respectively were embedded in polymethylmethacrylate (Technovit 3040, Heraeus Kulzer, Wehrheim, Germany) in order to allow for fixation to the apparatus used for biomechanical testing. Specimens were then sealed in triple plastic bags and stored at $-20^{\circ} \mathrm{C}$. Prior to testing, the specimens were thawed overnight at $4^{\circ} \mathrm{C}$.

Biomechanical testing was performed by means of a custom frame able to apply pure unconstrained moments in the three anatomical planes ${ }^{30)}$. ROM and neutral zone (NZ) were acquired by means of an optoelectronic motion analysis system (Vicon MX13, Vicon Motion Systems Ltd., Oxford, UK) and a 6 component load cell mounted above the specimen. Pure moments of $7.5 \mathrm{Nm}$ were applied in flexion-extension, left and right lateral bending, left and right axial rotation ${ }^{31)}$. For each motion, 3.5 loading-unloading cycles were performed, the first two of which were used for preconditioning whereas the latter full cycle was considered for data analysis.

An incremental protocol was used to investigate various types of decompression surgeries (Figs. 1 and 2). First, all specimens were tested in intact conditions. Then, a unilateral laminotomy for bilateral decompression (UNI-1L) was performed at L3-L4 on the left side of all specimens ${ }^{7)}$ and flexibility testing was repeated. Subsequently, specimens were randomly divided in two groups ( $\mathrm{n}=3$ for each group), in which a more extensive decompression was conducted at L3-L4 and at L3-L5 respectively. In the first group, a laminectomy with preservation of the facet joints and a laminar bony bridge, as routinely performed at the Department of Neurosurgery of Humanitas Clinical and Research Center, Rozzano, Italy, was performed at L3-L4 (BI-1L), followed by fixation with pedicle screws and titanium rods at L3-L4 (3LOCK, Sintea Plustek Srl, Assago, Italy). According to this surgical technique, only a

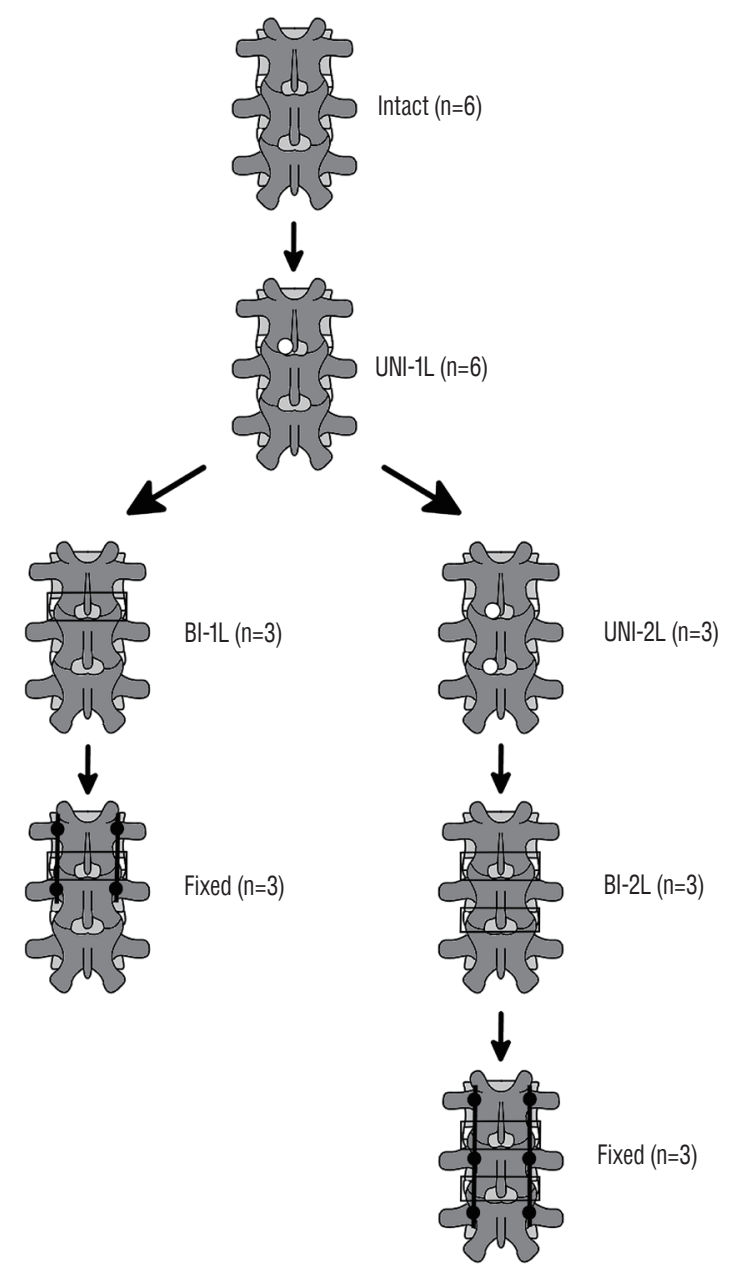

Fig. 2. Schematic representation of the incremental protocol of decompression and fixation. Intact : specimens before decompression, UNI-1L : after unilateral laminotomy for bilateral decompression at L3-L4, UNI-2L : after unilateral laminotomy for bilateral decompression at L3-L5, BI-1L : after bone-preserving laminectomy at $\mathrm{L} 3-\mathrm{L} 4, \mathrm{BI}-2 \mathrm{~L}$ : after bone-preserving laminectomy at L3-L5, fixed : after posterior fixation with pedicle screws and titanium rods at L3-L4 or L3-L5. 
bony bridge with a relatively small height and thickness is preserved in the vertebral arch, allowing for a conspicuous enlargement of the spinal canal (Fig. 3).

In the second group, the unilateral laminotomy for bilateral decompression was first extended at L4-L5 (UNI-2L) and followed by the bone-preserving laminectomy procedure described above at both levels (BI-2L). The specimens were then treated with pedicle screws and rods at L3-L5. Flexibility testing was repeated after each decompression and fixation step. A neurosurgical microdrill (Primado 2, NSK-Nakanishi Inc., Kanuma, Japan) was used to perform all decompression procedures.

After assessing the non-normality of the samples by means of the Shapiro-Wilk test, the Wilcoxon signed-rank test was used to assess if the unilateral laminotomy at L3-L4 induced
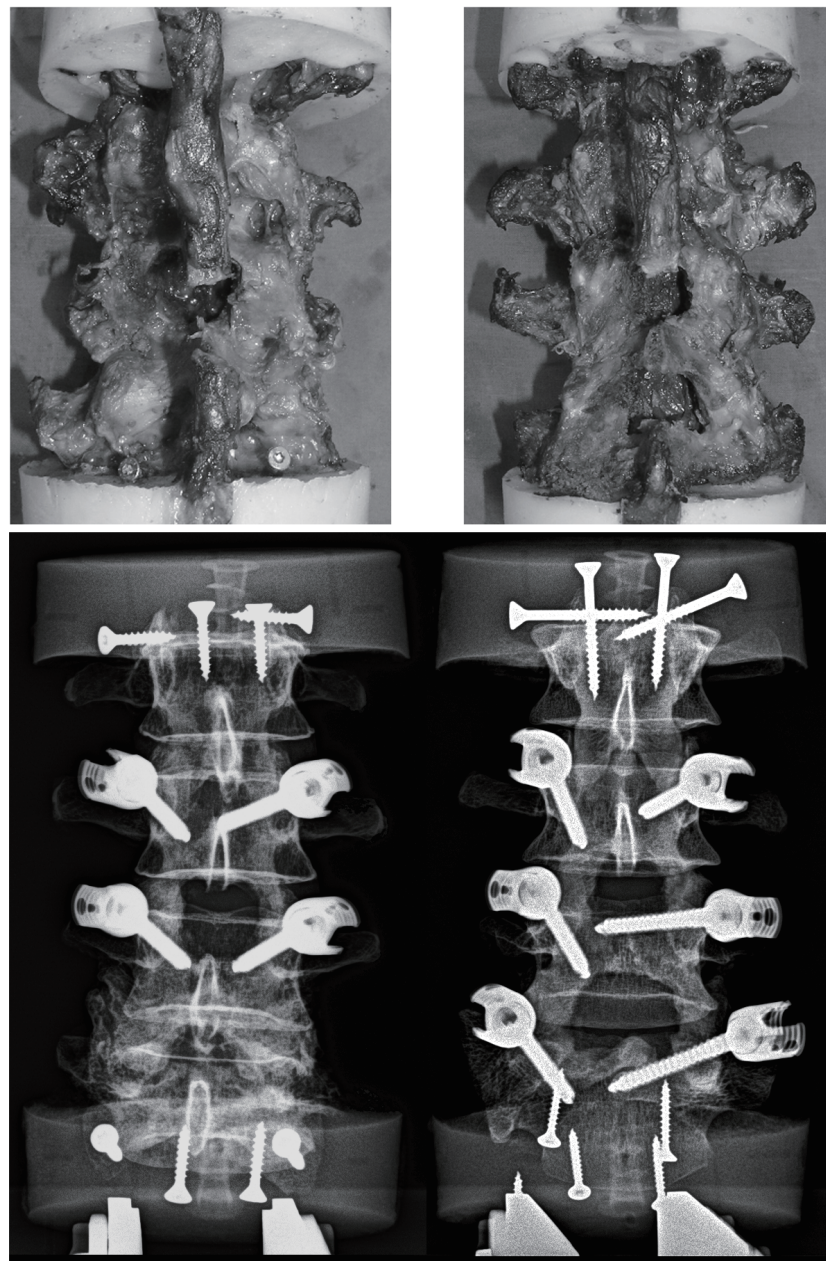

Fig. 3. Photographs (top) and anteroposterior radiographic projections (bottom) of specimens after bone-preserving laminectomy at L3-L4 (left) and L3-L5 (right). a statistically significant alteration of ROM and NZ with respect to the intact condition ${ }^{31}$. No statistics were performed on the other decompression procedures due to the limited number of specimens; results were therefore only qualitatively analyzed. The study has been approved by the Ethics Committee of Ulm University (IRB No. 72/16).

\section{RESULTS}

UNI-1L at L3-L4 had a minor influence on the flexibility of the lumbar spine in all motion planes (Fig. 4). In flexion-extension, the median ROM increased by $8 \%$ without statistical significance $(p=0.17$ ), whereas the increase of the median NZ was significant $(+31 \%, p=0.04)$. In left lateral bending, significant increases were revealed for both the median ROM and the NZ after unilateral laminotomy $(+4 \%[p=0.02]$ and $+29 \%$
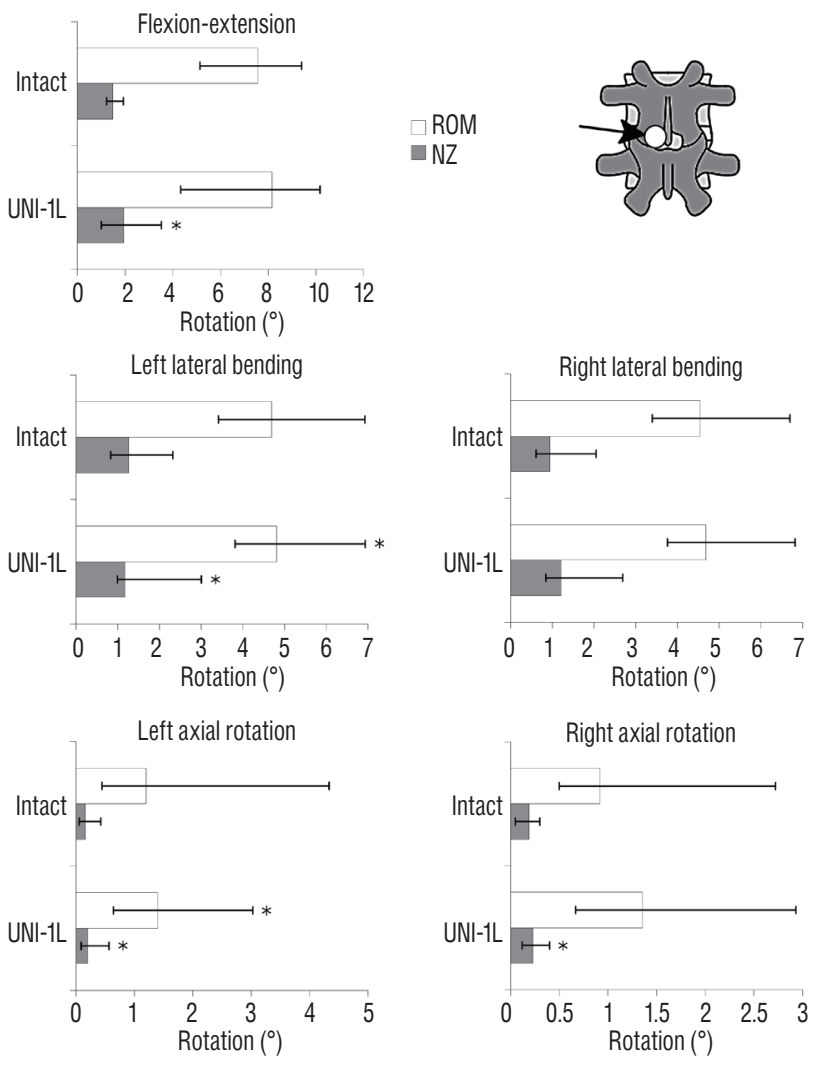

Fig. 4. Statistical comparison of the range of motion (ROM) and neutral zone (NZ) between the intact specimens and those subjected to unilateral laminotomy for bilateral decompression at L3-L4 (UNI-1L), in flexionextension, left and right lateral bending, left and right axial rotation. *A statistically significant difference $(p<0.05)$ between the intact and the decompressed condition $(n=6)$. 
[ $p=0.02]$, respectively), whereas in right lateral bending no significant alterations were detected. Similarly, left axial rotation showed a significant destabilization both in the median ROM $(+47 \%, p=0.01)$ and NZ $(+20 \%, p=0.02)$, whereas in the opposite direction only a significant NZ increase was found $(+26 \%$, $p=0.03$ ). Based on these results, unilateral laminotomy for bilateral decompression had a greater destabilization effect in ipsilateral rather than contralateral rotations.

Extending the decompression to a bone-preserving laminectomy (BI-1L) at L3-L4 did not determine major changes in spinal flexibility (Fig. 5). Nevertheless, in flexion-extension two of the three specimens had a marked ROM increase (+9\% and $+81 \%$ ), whereas the latter specimen was negligibly affected; NZs showed a similar trend. In lateral bending and axial rotation, both ROM and NZ were only marginally altered. Posterior fixation with pedicle screws and titanium rods strongly decreased ROMs and NZs in all cases.
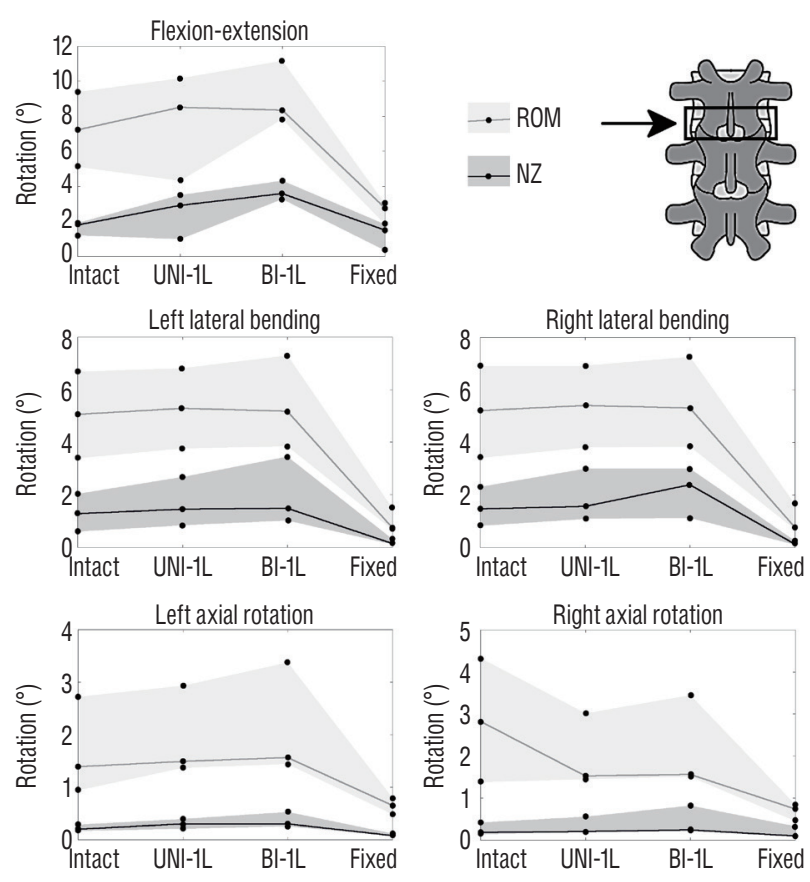

Fig. 5. Changes in the range of motion (ROM) and neutral zone (NZ) of the L3-L4 motion segment (indicated by the arrow) due to incremental decompression performed at the same level, in flexion-extension, left and right lateral bending, left and right axial rotation. The gray areas indicate the range between the maximum and minimum ROM and NZ among the three specimens, whereas the solid lines indicate the median value. Intact : specimen before decompression, UNI-1L : after unilateral laminotomy for bilateral decompression at $\mathrm{L} 3-\mathrm{L} 4, \mathrm{BI}-1 \mathrm{~L}$ : after bone-preserving laminectomy at $\mathrm{L} 3-\mathrm{L} 4$, fixed : after posterior fixation with pedicle screws and titanium rods at $\mathrm{L} 3-\mathrm{L} 4$.
Two-level decompression induced slightly higher changes in the spine flexibility (Figs. 6 and 7). The most evident effects of extending the unilateral laminotomy to L3-L5 were an increase of both ROM and NZ in flexion-extension at L4-L5, and of the ROM in axial rotation at the same level. The twolevel bone-preserving laminectomy (BI-2L) exacerbated the same effects. At the upper decompressed level (L3-L4), the effects of double-level decompression procedures were rather minor. Again, posterior fixation determined a major decrease of both ROM and NZ in all cases.

\section{DISCUSSION}

Bone-preserving decompression procedures are nowadays widely used for the treatment of lumbar stenosis and are pro-
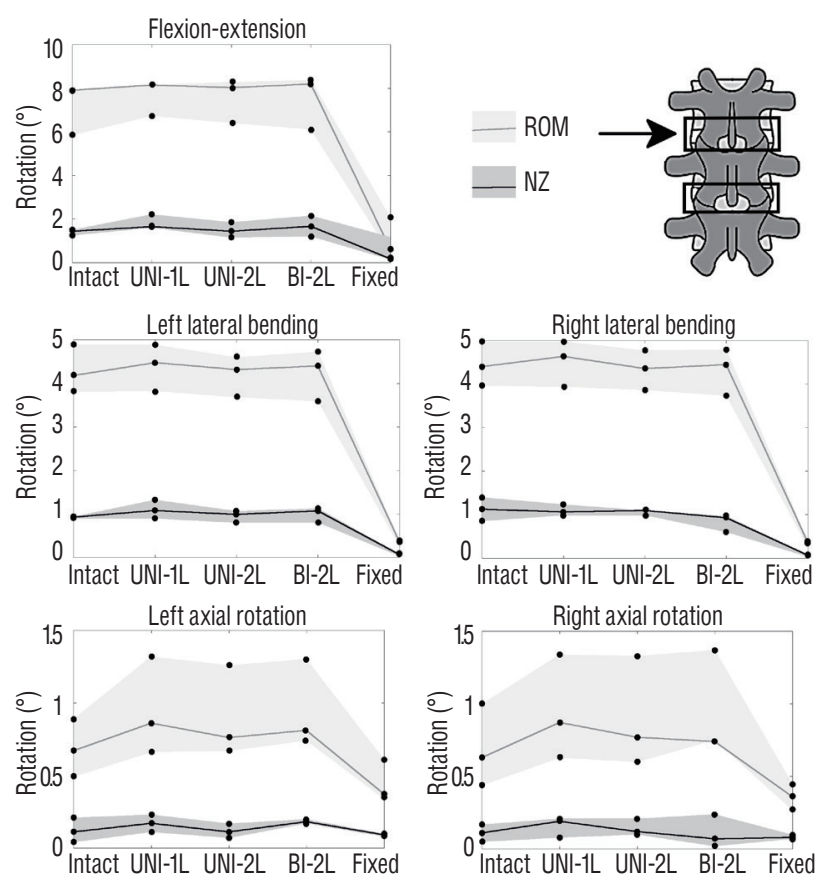

Fig. 6. Changes in the range of motion (ROM) and neutral zone (NZ) of the L3-L4 motion segment (indicated by the arrow) due to incremental decompression performed at the L3-L4 and L4-L5, in flexion-extension, left and right lateral bending, left and right axial rotation. The gray areas indicate the range between the maximum and minimum ROM and NZ among the three specimens, whereas the solid lines indicate the median value. Intact : specimen before decompression, UNI-1L : after unilateral laminotomy for bilateral decompression at L3-L4, UNI-2L : after unilateral laminotomy at L3-L5, BI-1L : after bone-preserving laminectomy at L3$L 5$, fixed : after posterior fixation with pedicle screws and titanium rods at L3-L5. 
gressively replacing the use of wide laminectomy ${ }^{25}$. In addition to the preservation of a higher degree of spine stability here investigated, less invasive techniques allow reducing operative time, minimizing blood loss as well as dural and radicular complications $s^{6,11)}$. The present in vitro study confirms that these techniques, in particular the unilateral laminotomy for bilateral decompression, are effective in preserving a physiological stability of the operated level, both in terms of ROM and NZ in all motion planes. This finding is in contrast with available data about wide laminectomy, which was found to determine an increase of the spinal flexibility after decompression $^{5,15,21)}$.

Spinal instability has been defined as an abnormal response to physiological loads, such as increased flexibility and $\mathrm{NZ}^{20}$. Natural instability, i.e., not related to a surgical intervention such as decompression, has been associated to an early stage
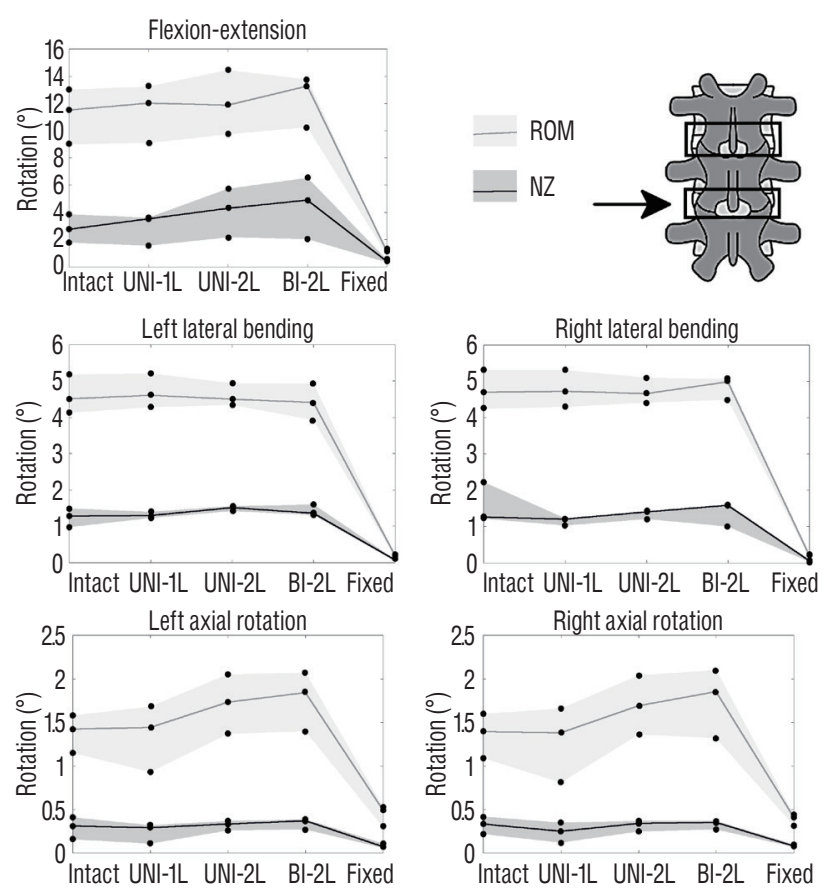

Fig. 7. Changes in the range of motion (ROM) and neutral zone (NZ) of the L4-L5 motion segment (indicated by the arrow) due to incremental decompression performed at the L3-L4 and L4-L5, in flexion-extension, left and right lateral bending, left and right axial rotation. The gray areas indicate the range between the maximum and minimum ROM and NZ among the three specimens, whereas the solid lines indicate the median value. Intact : specimen before decompression, UNI-1L : after unilateral laminotomy for bilateral decompression at L3-L4, UNI-2L : after unilateral laminotomy at L3-L5, BI-1L : after bone-preserving laminectomy at L3$L 5$, fixed : after posterior fixation with pedicle screws and titanium rods at L3-L5. of disc degeneration ${ }^{17)}$. Disc disruptions and tears have been indicated as the main determinants for spinal instability associated to degeneration ${ }^{17)}$, even if contrasting results have been reported $^{24)}$. Furthermore, biomechanical studies showed that dehydration of the nucleus pulposus due to the loss of proteoglycan content can also be responsible of increased flexibili$t^{33)}$. It should be noted that these degenerative changes are frequently occurring in the elderly patients requiring surgical decompression, and can therefore aggravate the effect of the iatrogenic lesion.

The study was based on a limited number of specimens $(n=6)$. Indeed, the subdivision in two subgroups further restricted the statistical validity of the study, as no statistical tests could be performed to evaluate the differences between UNI-1L, UNI-2L and laminectomy procedures. We ought to mitigate the relevance of this limitation by selecting specimens harvested from subjects with similar age, body weight and having minimal degenerative signs, thus reducing the variability among specimens. Nevertheless, a certain degree of variability among the specimens cannot be avoided and may have an influence on the results.

It should be noted that all specimens had no or minimal signs of lumbar stenosis, and are therefore not well representative of patients which may be subjected to decompression surgery. This choice was dictated by the fact that specimens selected to have lumbar stenosis are usually harvested from elderly subjects and exhibit other degenerative or pathological changes, in terms of both morphology and tissue composition, in combination to the canal narrowing, which may act as confounding factors. Selecting a homogeneous group of relatively young and healthy specimens appeared therefore the most adequate option to investigate the specific biomechanical effect of the decompression techniques.

Aside from intervertebral discs and spinal ligaments, no soft tissues were preserved in the specimens and thus included in the mechanical tests, following consolidated procedures extensively used in in vitro tests of human specimens ${ }^{31}$. Indeed, testing specimens including muscles and skin would be technically challenging and is not a viable option for most spine testers and loading systems. Nevertheless, the limited size of the surgical access is an essential part of a minimally invasive procedure, and an experimental evaluation of the biomechanical influence of the size of the surgical access itself on the risk of instability would be valuable. 
The experiments were carried out by using a pure moment loading protocol ${ }^{31}$, which did not allow for the investigation of the influence of decompression and fixation on the adjacent segments ${ }^{28)}$. Although other studies reported alterations of the adjacent levels after application of a pure moment protocol $^{4)}$, it was demonstrated that they may be attributed to inaccuracies of the testing apparatus rather than to biomechanical reasons related to the surgical procedures ${ }^{28}$. Indeed, the evaluation of adjacent segment effects would be clinically valuable, especially after instrumented fixation, but no established protocols to correctly measure them in vitro currently exist.

Despite the limitations mentioned above, the present results provide the base for several clinically relevant remarks. First, bilateral decompression performed through a unilateral laminotomy had a minor influence on the stability of the operated level and can therefore be deemed as weakly relevant as a possible cause of iatrogenic instability. Indeed, statistically significant increases of both ROM and NZ have been measured in lateral bending and axial rotation in the ipsilateral direction, but the median magnitude of the increases could be safely judged as marginal from a clinical point of view. In ipsilateral axial rotation, a marked relative ROM increase $(+47 \%)$ was found, but it should be noted that it corresponded to an average destabilization of $0.4^{\circ}$ in absolute terms. The same consideration applies to the statistically significant increases of NZ, which are in absolute terms practically negligible. These findings are in good agreement with the finite element predictions of Ivanov and coworkers ${ }^{14)}$, who also reported a marginal destabilization after minimally invasive decompression. The authors however showed a significant stress gain in the remaining portion of the neural arch, which may lead to fatigue fractures postoperatively. Due to the experimental nature of the present study, this remark could not be confirmed nor refuted, but it should indeed be taken into account in future investigations.

Despite statistical analysis could not be performed due to the limited number of specimens, the bone-preserving laminectomy did not seem to create a marked instability with respect to both the intact state and unilateral laminotomy for bilateral decompression. These findings are in contrast with previous biomechanical studies, in which laminectomy both with or without facetectomy generated a significant decrease of the spine stiffness at the operated level ${ }^{3,4,16,19,32)}$. The bony bridge preserved in this technique therefore had a beneficial effect in terms of spine stability. Therefore, from a purely biomechanical point of view, this surgical technique may be advantageous over wide laminectomy for cases in which a unilateral approach is insufficient. However, prior to select any decompression technique including bone-preserving ones, we recommend performing flexion-extension X-rays pre-operatively in order to assess the spine stability and thus to prevent the aggravation of already existing instabilities.

Testing of two-level procedures revealed that the biomechanical effect of both unilateral laminotomy and laminectomy was higher at L4-L5 than at L3-L4. Such result may be expected based on the higher flexibility of the native lower lumbar spine in comparison to higher levels, related to the higher disc height and other anatomical characteristics such as facet orientation ${ }^{29)}$. It should be noted that the specimens used in this study had minimal degenerative signs at both L3L4 and L4-L5, thus an association between possible degenerative changes and the higher effect of decompression at L4-L5 should be excluded. Taking into account the limitations of the study, it appears that a decompression at L4-L5 may be in principle more critical in terms of risk of iatrogenic destabilization of the operated level.

\section{CONCLUSION}

In summary, in vitro testing of human lumbar specimens revealed that unilateral laminotomy for bilateral decompression induced a minor destabilization at the operated level in terms of ROM and NZ in the three motion planes. Bone-preserving laminectomy also had a limited biomechanical effect. In absence of confounding factors which may be relevant in some specific clinical cases (e.g., clinical instability, spondylolisthesis) and taking into account that the clinical outcome of any decompression procedure does not depend only on biomechanical factors, both techniques appeared to be safe from a biomechanical point of view.

\section{CONFLICTS OF INTEREST}

No potential conflict of interest relevant to this article was reported. 


\section{INFORMED CONSENT}

This type of study does not require informed consent.

\section{- Acknowledgements}

The work has been funded by the Italian Ministry of Health (project GR-2011-02351464). Pedicle screws and rods have been provided for free by Sintea Plustek Srl (Assago, Italy). The microdrill has been loaned by Tekmed Instruments Spa (Rozzano, Italy).

\section{References}

1. Airaksinen $O$, Herno $A$, Turunen $V$, Saari $T$, Suomlainen 0 : Surgical outcome of 438 patients treated surgically for lumbar spinal stenosis. Spine (Phila Pa 1976) 22 : 2278-2282, 1997

2. Allen $B L \mathrm{Jr}$, Tencer $A F$, Ferguson $R L$ : The biomechanics of decompressive laminectomy. Spine (Phila Pa 1976) 12 : 803-808, 1987

3. Bisschop A, Kingma I, Bleys RL, van der Veen AJ, Paul CP, van Dieën JH, et al. : Which factors prognosticate rotational instability following lumbar laminectomy? Eur Spine J 22 : 2897-2903, 2013

4. Bisschop A, van Engelen SJ, Kingma I, Holewijn RM, Stadhouder A, van der Veen AJ, et al. : Single level lumbar laminectomy alters segmental biomechanical behavior without affecting adjacent segments. Clin Biomech (Bristol, Avon) 29 : 912-917, 2014

5. Bisschop A, van Royen BJ, Mullender MG, Paul CP, Kingma I, Jiya TU, et al. : Which factors prognosticate spinal instability following lumbar laminectomy? Eur Spine J 21 : 2640-2648, 2012

6. Carreon LY, Puno RM, Dimar JR 2nd, Glassman SD, Johnson JR : Perioperative complications of posterior lumbar decompression and arthrodesis in older adults. J Bone Joint Surg Am 85-A : 2089-2092, 2003

7. Costa F, Sassi M, Cardia A, Ortolina A, De Santis A, Luccarelli G, et al. : Degenerative lumbar spinal stenosis: analysis of results in a series of 374 patients treated with unilateral laminotomy for bilateral microdecompression. J Neurosurg Spine 7 : 579-586, 2007

8. diPierro CG, Helm GA, Shaffrey $\mathrm{Cl}$, Chadduck JB, Henson SL, Malik $J M$, et al. : Treatment of lumbar spinal stenosis by extensive unilateral decompression and contralateral autologous bone fusion: operative technique and results. J Neurosurg 84 : 166-173, 1996

9. Fox MW, Onofrio BM, Hanssen AD : Clinical outcomes and radiological instability following decompressive lumbar laminectomy for degenerative spinal stenosis: a comparison of patients undergoing concomitant arthrodesis versus decompression alone. J Neurosurg 85 : 793-802, 1996

10. Fritz $\mathrm{JM}$, Erhard RE, Vignovic $\mathrm{M}$ : A nonsurgical treatment approach for patients with lumbar spinal stenosis. Phys Ther 77 : 962-973, 1997

11. Fujita T, Kostuik JP, Huckell CB, Sieber AN : Complications of spinal fu- sion in adult patients more than 60 years of age. Orthop Clin North Am 29 : 669-678, 1998

12. Grob D, Humke T, Dvorak J : Degenerative lumbar spinal stenosis. Decompression with and without arthrodesis. J Bone Joint Surg Am 77 : 1036-1041, 1995

13. Iguchi T, Kurihara A, Nakayama J, Sato K, Kurosaka M, Yamasaki K : Minimum 10-year outcome of decompressive laminectomy for degenerative lumbar spinal stenosis. Spine (Phila Pa 1976) 25 : 1754-1759, 2000

14. Ivanov A, Faizan A, Sairyo K, Ebraheim N, Biyani A, Goel VK : Minimally invasive decompression for lumbar spinal canal stenosis in younger age patients could lead to higher stresses in the remaining neural arch -- a finite element investigation. Minim Invasive Neurosurg 50 : 18-22, 2007

15. Kato Y, Panjabi MM, Nibu K : Biomechanical study of lumbar spinal stability after osteoplastic laminectomy. J Spinal Disord 11 : 146-150, 1998

16. Katz JN, Lipson SJ, Larson MG, McInnes JM, Fossel AH, Liang MH : The outcome of decompressive laminectomy for degenerative lumbar stenosis. J Bone Joint Surg Am 73 : 809-816, 1991

17. Kirkaldy-Willis WH, Farfan HF : Instability of the lumbar spine. Clin Orthop Relat Res (165) : 110-123, 1982

18. Nakai O, Ookawa A, Yamaura I : Long-term roentgenographic and functional changes in patients who were treated with wide fenestration for central lumbar stenosis. J Bone Joint Surg Am 73 : 1184-1191, 1991

19. Phillips FM, Tzermiadianos MN, Voronov LI, Havey RM, Carandang G, Renner SM, et al. : Effect of the total facet arthroplasty system after complete laminectomy-facetectomy on the biomechanics of implanted and adjacent segments. Spine J 9 : 96-102, 2009

20. Pope $M H$, Panjabi $M$ : Biomechanical definitions of spinal instability. Spine (Phila Pa 1976) $10:$ 255-256, 1985

21. Quint $U$, Wilke $H J$, Löer $F$, Claes $L$ : Laminectomy and functional impairment of the lumbar spine: the importance of muscle forces in flexible and rigid instrumented stabilization--a biomechanical study in vitro. Eur Spine J 7 : 229-238, 1998

22. Silvers HR, Lewis PJ, Asch HL : Decompressive lumbar laminectomy for spinal stenosis. J Neurosurg 78 : 695-701, 1993

23. Simotas AC, Dorey FJ, Hansraj KK, Cammisa F Jr : Nonoperative treatment for lumbar spinal stenosis. clinical and outcome results and a 3-year survivorship analysis. Spine (Phila Pa 1976) 25 : 197-203, 2000

24. Tanaka N, An HS, Lim TH, Fujiwara A, Jeon CH, Haughton VM. The relationship between disc degeneration and flexibility of the lumbar spine. Spine J 1 : 47-56, 2001

25. Thomé $C$, Zevgaridis $D$, Leheta $O$, Bäzner $H$, Pöckler-Schöniger $C$, Wöhrle $J$, et al. : Outcome after less-invasive decompression of lumbar spinal stenosis: a randomized comparison of unilateral laminotomy, bilateral laminotomy, and laminectomy. J Neurosurg Spine 3 : 129-141, 2005

26. Tuite GF, Stern JD, Doran SE, Papadopoulos SM, McGillicuddy JE, Oyedijo DI, et al. : Outcome after laminectomy for lumbar spinal stenosis. Part I: clinical correlations. J Neurosurg 81 : 699-706, 1994 
27. Verbiest $\mathrm{H}$ : A radicular syndrome from developmental narrowing of the lumbar vertebral canal. J Bone Joint Surg Br 36-B : 230-237, 1954

28. Volkheimer D, Malakoutian M, Oxland TR, Wilke WJ : Limitations of current in vitro test protocols for investigation of instrumented adjacent segment biomechanics: critical analysis of the literature. Eur Spine J 24 : 1882-1892, 2015

29. White $A A$, Panjabi MM : Clinical biomechanics of the spine, ed 2. Philadelphia : Lippincott Williams \& Wilkins, 1990

30. Wilke $H J$, Claes $L$, Schmitt $H$, Wolf $S:$ A universal spine tester for in vitro experiments with muscle force simulation. Eur Spine J 3 : 91-97, 1994

31. Wilke HJ, Wenger K, Claes L : Testing criteria for spinal implants: recommendations for the standardization of in vitro stability testing of spinal implants. Eur Spine J 7 : 148-154, 1998

32. Zander $T$, Rohlmann A, Klöckner C, Bergmann $G$ : Influence of graded facetectomy and laminectomy on spinal biomechanics. Eur Spine J 12 : 427-434, 2003

33. Zhao F, Pollintine $P$, Hole BD, Dolan $P$, Adams MA : Discogenic origins of spinal instability. Spine (Phila Pa 1976) 30 : 2621-2630, 2005 